\title{
The effect of LLLT on bone metabolism in children with severe cerebral palsy (a secondary publication)
}

\author{
Yoshimi Asagai \\ Department of Orthopedic Surgery, Shinano Handicapped Children's Hospital
}

\begin{abstract}
Background and aims: It is said that the average frequency of bone fracture in hospitalized children with severe cerebral palsy (unable to remain seated) is $1 \%$ ( 0.2 to $2.0 \%$ ). Cerebral palsy patients' bones are known to be vulnerable to fracture, and refractory bone atrophy may be observed. However, the effect of low level laser therapy (LLLT) on bone density or bone metabolism has not been fully investigated. In recent years, tests for bone density or bone metabolism markers have become available.

Material and methods: In this study, we evaluated changes in bone density and bone metabolism markers in 4 children with severe cerebral palsy who underwent LLLT for an average of 22 days.

Results: B-ALP, a marker of ossification, increased 1 month after the start of irradiation in 3 of the 4 subjects and returned to a level close to the pre-irradiation level 2 months after the start of irradiation. In the remaining subjects in whom B-ALP failed to increase, B-ALP had been low before irradiation. Urinary N-terminal telopeptide (NTx) levels, a marker of bone resorption, decreased in 3 of the 4 subjects after the start of irradiation and remained low even 10 months later. Serum NTx levels tended to decrease in 3 of the 4 subjects. The levels of serum NTx/Crea, Deoxy-Pyridinoline (DPd) and DPd/Crea (DPd/Crea) also decreased in 3 of the 4 subjects. Transient decreases in intact parathyroid hormone (PTH) levels were observed in all 4 cases. Changes were particularly apparent in 2 cases: one with high NTx levels, which showed enhanced bone resorption, and one with high PTH levels, probably due to a vitamin D (VitD) deficiency. Although the metacarpal bone density measured by DIP was found to be lower than in normal children, there were no changes due to LLLT.

Conclusion: These results suggest that LLLT has a positive influence on bone metabolism in that it temporarily increases bone formation and suppresses bone resorption while also tending to improve secondary hyperparathyroidism caused by VitD deficiency.

Enhanced bone resorption in the case with high NTx levels was noteworthy, together with marked changes in the case with high PTH levels due to VitD deficiency. These positive influences on bone metabolism merit attention as potential new indications of LLLT.
\end{abstract}

Key words: low level laser therapy - children with severe cerebral palsy • bone metabolism • pathological fracture

\section{Introduction}

Pathological fractures in children with severe cerebral palsy do not require extreme external force and may occur from routine daily care or from episodes of convulsions. If one is not aware of the pathological fractures, such fractures can be mistaken for medical accidents and lead to malpractice suits. Concerning bone

\section{Addressee for Correspondence:}

Yoshimi Asagai MD

Director, Shinano Handicapped Children's Hospital, 6525-1

Shimosuwa, Suwagun, Nagano, Japan 393-0093.

Tel: +81-266-27-8414 Fax: +81-266-27-7936

E-mail:asagai@shinano-iryo.or.jp metabolism and the pathological fractures of children with severe cerebral palsy, a study "A study on the long-term prognosis and functional improvement, pathology of severe mentally and physically handicapped children" 1) reported that bone formation is less while bone resorption is greater in children with severe mental and physical handicaps when compared to normal children. Recently, the efficacy of bisphosphonates administration in elderly high turnover type osteoporosis patients showing high levels of bone resorption markers is well known. However, it has

Received date: July 11th, 2014

Accepted date: November 5th, 2014

Laser Therapy 23.4: 243-247 
been experimentally proven that even such suppressants of bone resorption do not help ossification at the diaphysis of long bones in patients whose resorption is due to lack of weight load stress. This means that bisphosphonates are useless for bedridden children with severe cerebral palsy ${ }^{2}$. As of now, there is no established treatment of severe osteoporosis where bone mineral density has decreased.

The effect of LLLT on bone metabolism has always attracted attention, however the subject has not been studied thoroughly due to the lack of an objective evaluation method. Akai has reported that under optimum conditions, LLLT may possibly reduce contracture related changes to bone and cartilage 3 ). Hiragami has reported of three-dimensional growth and proliferation of fibroblasts cultured on an artificial bone scaffold when irradiated with laser at certain settings ${ }^{4)}$. Barushuka et al. reported that the amount of osteoblast specific alkaline phosphatase appearing during bone regeneration was significantly greater in the laser irradiated group ${ }^{5)}$, while Yamada reported that laser irradiation promoted osteoblast growth and earlier calcification ${ }^{6}$.

In this study we investigated the effect of LLLT on the bone metabolism in children with severe cerebral palsy.

\section{Materials and methods}

Four cases of severe pediatric spastic type cerebral palsy were enrolled in this study. All patients were unable to maintain a sitting position. The age of the patients ranged from 11 17 years-old, with an average of 13 years; 3 patients were male and 1 was female. The laser device used for the study was a GaAlAs diode laser JQ305 (Minato Medical Science Co. LTD., Osaka, Japan), wavelength $810 \mathrm{~nm}$, output $100 \mathrm{~mW}$, continuous wave emission with an irradiated area of $1.04 \mathrm{~mm}^{2}\left(0.0104 \mathrm{~cm}^{2}\right)$, power density of $9.61 \mathrm{~W} / \mathrm{cm}^{2}$, and energy density per point of $9.61 \mathrm{~W} / \mathrm{cm}^{2}$ x $60 \mathrm{sec}$. The laser therapy was given once a day, five days per week; the period during which laser therapy was delivered ranged from 18 24 days, with an average of 22 days. The points of laser irradiation were those used to alleviate the muscle spasticity of cerebral palsy patients; the points were at the neck, 4 points along the thoracic and lumbar paraspinal musculature, the root of the tibial nerve, and over the gôkoku and inpô acupuncture points for a total of 16 points for both sides of the body. Each point was irradiated with the laser for 1 minute to give a total irradiation time of 16 minutes per session 7,8 ). Urinalysis and blood sample analysis were performed prior to the laser irradiation and at 1, 2, 4, 10 and 24 months after laser irradiation and the following were examined: Type 1 collagen cross-linked N-telopeptide (NTx)/crea, DeoxyPyridinoline (DPd), DPd/crea, serum NTx, and urine NTx were measured as bone resorption markers while bone specific alkaline phosphatase (B-ALP), measured with an enzyme immuno-assay, and osteocalcin were used as markers for bone formation. Parathyroid hormone levels (PTH-intact) were measured as an index for secondary hyperparathyroidism due to vitamin D (VitD) deficiency. Bone densities were measured from X-ray films of the third metacarpal bone of the left hand, using a digital image processing (DIP) method.

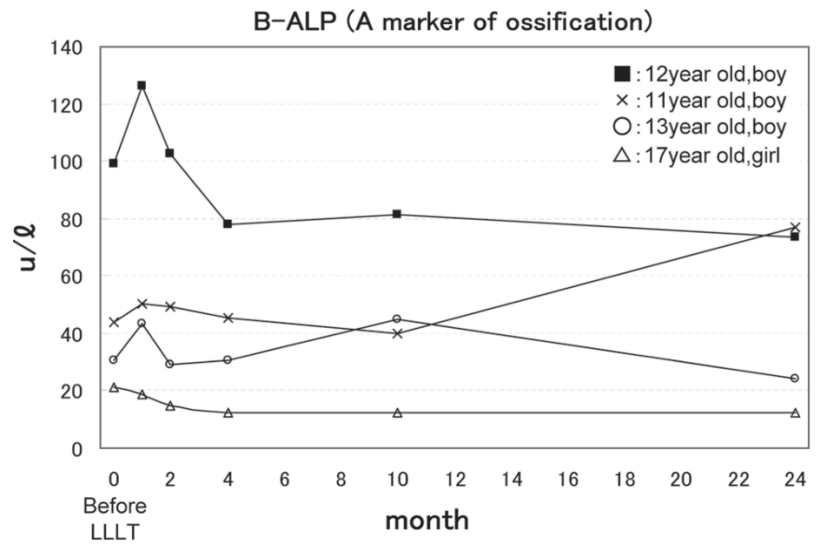

Fig. 1: LLLT tended to stimulate ossification temporarily.

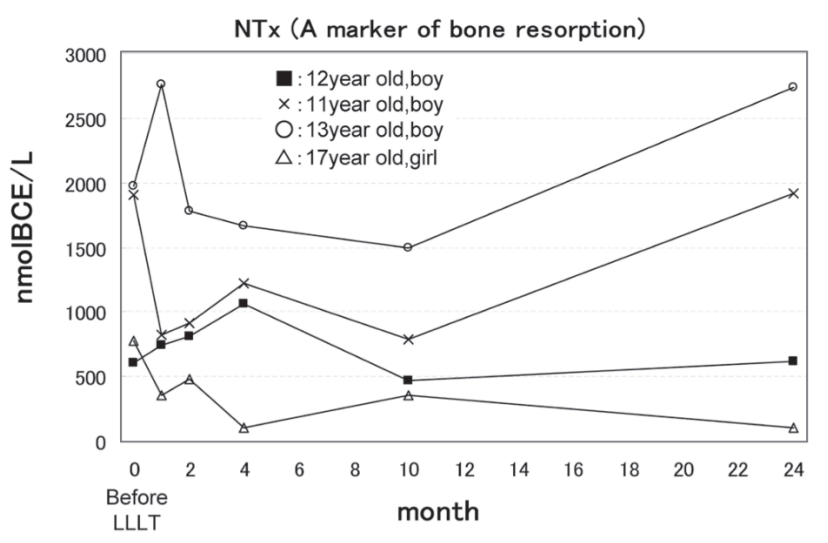

Fig. 2: LLLT tended to suppress bone resorption temporarily. 


\section{Results}

Of the 4 severe cerebral palsy patients, the ossification marker B-ALP increased in 3 patients at 1 month after laser irradiation. The increase was transient in that the level of B-ALP fell to pre-treatment levels at 2 months and no change was seen from then and up to the $24^{\text {th }}$ month. As for the case where no increase in B-ALP was seen, the baseline B-ALP level was low in the first place (Figure 1). Urine NTx, a bone resorption marker, decreased in 2 patients at 1 month, in 3 patients at 2 months and in all 4 patients at 10 months. However in 3 of the 4 patients, the level of urine NTx returned to the pre-treatment level at 24 months (Fig. 2). Serum NTx showed tendencies to decrease after laser irradiation in 3 of the 4 patients. NTx/crea, DPd, and $\mathrm{DPd} /$ crea also decreased after laser irradiation in 3 of the 4 patients. The levels of PTH-intact decreased at 4 months in 3 of the 4 patients, and similar tendencies were still seen at 24 months. The changes were greater in 2 patients; one being a case with high NTx levels and accelerated bone resorption (Figure 2) and the other being a case with high PTH levels probably due to VitD deficiency (Figure 3). The increase or decrease which followed laser irradiation did not exceed normal levels. The bone mineral densities of the 4 patients measured using DIP of the third metacarpal bone of the left hand were less when compared to normal healthy children ${ }^{9}$ ). There were tendencies towards a transient increase in bone density following LLLT, but no apparent change was seen (Figure 4).

\section{Discussion}

The reasons why pathological fractures are seen in children with severe cerebral palsy are as follows: they cannot maintain a posture of sufficient weight load to stimulate bone growth; the anticonvulsants prescribed to those patients disrupt normal VitD metabolism; lack of exposure to sunlight; malnutrition; and hormonal disruption.

Indices for the risk of pathological fractures are radiographic findings, bone mineral density, bone turnover markers, vitamin $\mathrm{K}$ (VitK) and undercarboxylated osteocalcin (ucOC), while indices of bone quality are the measurements of overexpression of pathologic crosslink compounds (e.g., pentosidine), and of homocysteine, 25-hydroxyvitamin D [25(OH)D] and parathyroid hormone (PTH). Recently, it has been noted that a decrease in $25(\mathrm{OH}) \mathrm{D}$ causes secondary hyperparathyroidism and increases in PTH are seen. However it also has been noted that high incidences of pathological fractures occur in those with decreased PTH levels despite the decrease of $25(\mathrm{OH}) \mathrm{D}$.

In terms of treatment, an LD-based (laser diodebased) system was used in the present study, but the new generation of light-emitting diodes (LEDs) has been attracting a lot of attention as an alternative phototherapeutic source. As with LDs, the energy emitted by near-infrared LEDs is known for its deep penetration into living tissue, and larger areas can be targeted compared to lasers. The application of LEDs for enhancement of wound healing and the prevention of

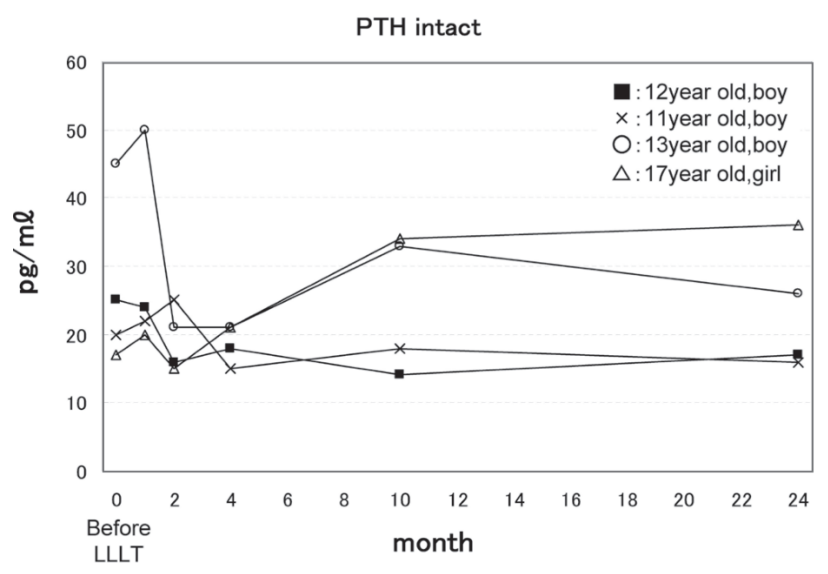

Fig. 3: LLLT tended to suppress PTH temporarily.

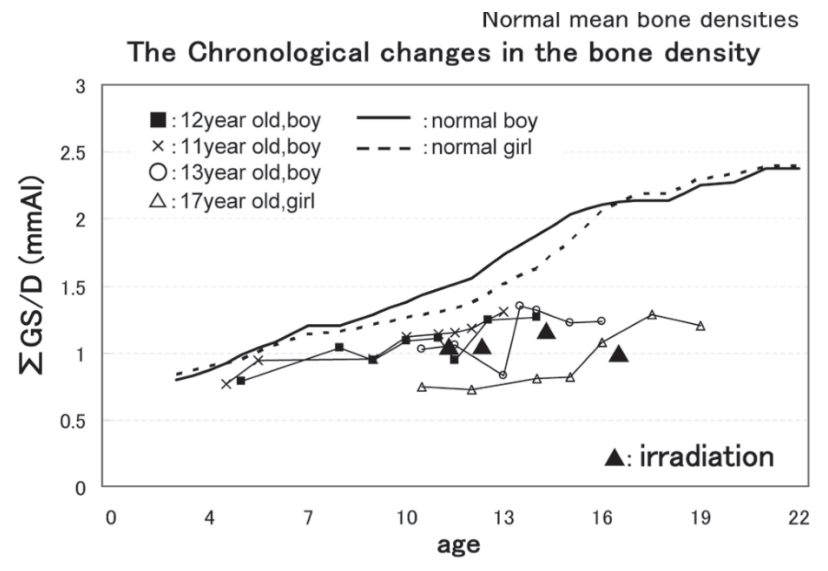

Fig. 4: The Chronological changes in the bone density. The black solid line indicates the normal mean bone densities of boys, and the black dotted line indicates the normal mean values of girls. 
muscle and bone atrophy for astronauts have been reported in United States National Aeronautics and Space Administration (NASA)-related literature ${ }^{10)}$. The indication of this interesting light source for acupuncture points need to be assessed in future studies.

Concerning bone mineral density measurement, we used the DIP method, the only method available at our institution. Therefore we were not able to directly evaluate the density of the femur which is the bone most susceptible to pathological fracture. However, in general, cerebral palsy patients have better movement of the upper limbs compared to the lower limbs. This should mean a low BMD of the upper limbs would denote an even lower BMD of the lower limbs and we believe that upper limb BMD is capable of being an indicator for lower limb bone atrophy.

The level of bone turn-over markers changes along with the age in children with severe cerebral palsy. Some markers demonstrate diurnal variations and as of yet no established normal value for the markers of healthy children exist. Further studies are warranted if the markers are to be used as objective indices.

VitD deficiency is involved with the pathological fractures in children with severe cerebral palsy and in some cases secondary hyperparathyroidism is seen. In Japan, the test for $25(\mathrm{OH}) \mathrm{D}$ is not covered by the National Health Insurance system, therefore we used PTH as a marker instead. In this investigation, PTHintact levels decreased transiently at 2 4months after laser irradiation in all 4cases. This was especially so in

\section{References}

1: Yoshino K, Kimura M, Maeoka Y, et al. (1996). Research concerning bone fragility of severe mentally and physically handicapped children. In " $A$ study on the long - term prognosis and functional improvement, pathology of severe mentally and physically handicapped children" Annual research report 1996, Psychiatric and neurological diseases research commission expenses. 152-165.

2: Sugiyama T, Taguchi T, Kawai S (2004). Spontaneous fracture and quality of life in cerebral palsy.Lancet. 364(28).

3: Akai M (1997). Effect of low level laser therapy on joint contracture; A possibility of modifying muscloskeletal tissues with biophysical factor. Sogo Rehabil, 25:315-319.

4: Barushka O, Yaakobi T, Oron U (1995). Effect of low energy laser irradiation on the process of bone the patient with secondary hyperparathyroidism due to VitD deficiency and this demonstrates that LLLT had a favorable effect on VitD metabolism. There is a possibility that the previously reported homeostatic effect of LLLT is also seen in the bone metabolism of children with severe cerebral palsy.

Given the optimistic results of the current study, albeit with its very small population, we would like to pursue this research further in a study with a larger patient population, using more bone turn-over markers, VitK and other markers for bone quality.

\section{Conclusions}

1. In this study we investigated the effect of LLLT on bone mineral density and bone metabolism in children with severe cerebral palsy.

2. The short term record showed no effect of LLLT on bone mineral density but a transient increase in bone formation and suppression of bone resorption were observed. A tendency towards the improvement of secondary hyperparathyroidism due to VitD deficiency was also seen.

3 . In the case of accelerated bone resorption manifesting a high NTx level and in the case of VitD deficiency demonstrating a high PTH level, both NTx and PTH decreased to within normal limits after laser irradiation.

4. LLLT had a favorable effect on bone metabolism, and could have a high potential to become a new treatment modality for osteoporosis.

repair in the rat tibia. Bone. 16:47-55.

5: Hiragami F, Kano Y (2002). Formation of HA mediated three-dimensional structures by mouse fibroblasts due to physical stimulation. Jpn J Rehabil Med. 39:229-235.

6: Yamada K (1991). Biological effects of low power laser irradiation on clonal osteoblastic cells (MC3T3-E1). J. Jpn. Orthop. Assoc. 65:787-799.

7: Asagai Y, Kanai H, Miura Y, Ohshiro T (1994). Application of low reactive-level laser therapy (LLLT) in the functional training of cerebral palsy patients. Laser Therapy. 6:195-201.

8: Asagai Y, Sujaritpong T, Tranvan L, Ohshiro T (2007). Assessment of changes in carotid blood flow following LLLT of the neck. Laser Therapy. 16:127-132.

9: Matsuoka H (1994). Study of skeletal maturation in 
Japanese children based on bone mineral density estimation part1 standard values of healthy Japanese from 3 to 18 years based on chronological and bone age. J Jpn Pediatr Soc. 98(7):13641373 .
10: Whelan HT, Buchmann EV, Whelan NT, Turner SG, et al. (2001). NASA light emitting diode medical applications: From deep space to deep sea. Space Tech. E App. Int'l Forum. 552:35-45.

\section{[Acknowledgements]}

Editor's Note: This paper was originally published in Japanese in The Journal of Japan Society for Laser Surgery and Medicine, Vol. 32-4:389-392, 2012, and has been specially translated for inclusion in Laser Therapy as an English Original Article. 